\title{
Piphat Mon in the Korat Way of Life: Creating Performing Arts Standards to Promote the Thai Music Profession
}

\author{
Samutcha Jeeranai ${ }^{1}$, Sittisak Jampadaeng ${ }^{1}$ \& Somkit Suk-Erb ${ }^{1}$ \\ ${ }^{1}$ The Faculty of Cultural Science, Mahasarakham University, Khamriang Sub-District, Kantarawichai District, \\ Maha Sarakham, Thailand \\ Correspondence: Samutcha Jeeranai, Ban Bing School, Moo 8, Chokchai Sub-district, Chokchai District, \\ Nakhon Ratchasima 30190, Thailand. E-mail: samutcha007@gmail.com
}

Received: April 29, 2013 Accepted: May 29, 2013 Online Published: August 1, 2013

doi:10.5539/ass.v9n10p198 URL: http://dx.doi.org/10.5539/ass.v9n10p198

\begin{abstract}
Piphat Mon is the traditional form of music of Burmese Mon people. Following Mon immigration into Thailand, the art form was adopted and adapted by Thais as a medium for entertainment and worship. In the current climate of globalization and the influence of western trends, representation of Piphat Mon has been reduced to funeral ceremonies in a small collection of Thai provinces. This research examines the historical background and current conditions of Piphat Mon in Nakhon Ratchasima Province, its primary location in Northeast Thailand, with the aim of creating a set of performing arts standards. The results of this research show that Piphat Mon has changed in light of social and cultural changes to Thailand since 1998. Nowadays there are a number of problems with artistic performances of Piphat Mon in Korat, particularly concerning the attitudes of the performers, the sound, the equipment and the location. Using the standards created during this study, a solution to these problems is provided and Piphat Mon in Nakhon Ratchasima will continue to be developed and maintained despite the popularity of Western music.
\end{abstract}

Keywords: Piphat Mon, performing arts, standards, creation, Thai music profession, Korat lifestyle

\section{Introduction}

Mon, or Raman, are an ancient civilization with unique and advanced culture and art. In Southeast Asia there is evidence that Mon people once had a national territory, customs, culture and traditions of their own. These have been inherited over time. This culture can be seen in both the past and the present, for example speech, writing, costume, music and dance. These customs that show traces of the ancient Mon civilization have been visible in the Thai landscape since the Dvaravati period and show the development of Mon people in the past.

The Mon civilization has expanded into the Thai territory with Burmese immigrants for over four hundred years. Immigration first occurred in the eras of Somdet Prathammaracha, Prachao Prasat Thong and Somdet Pra Narai Maharat and has continuously occurred throughout the Ayutthaya, Thonburi and Rattanakosin periods of Thai history. The reason for emigration from Burma was fighting between the Burmese and Mon people. The general name for the Mon people who emigrated before and during the Thonburi period is 'Old Mon'. The general name for those who emigrated during the Rattanakosin period is 'New Mon'.

The areas most densely populated with Mon communities are in Sam Khok District, Pathum Thani Province, Pak Kret District, Nonthaburi Province and along the banks of the Tha Chin and Mae Klong Rivers, such as Ban Pong and Photharam Districts, Ratchaburi Province. Aside from these places, Mon communities are scattered across various provinces in Central Thailand, including Samut Sakhon, Samut Songkhram, Suphanburi, Phetchaburi, as well as Nakhon Sawan, Lamphun and Lampang Provinces in Northern Thailand and Nakhon Ratchasima Province in Northeastern Thailand (Pinpat, 2003, p. 1-3).

From the research of various commentators regarding the birth of Piphat Mon, it has been found that Piphat Mon, the music of Mon people, entered Siamese society from the Ayutthaya period. Examination of the evidence suggests that the music came to Thailand no earlier than the reign of Somdet Phra Narai Maharat. Records were written by foreign priests who came with diplomatic envoys to seek audience with Somdet Phra Narai Maharat detailing the performances of Siamese, Malay, Laos and Mon people. These records described alternate Mahori (Thai string orchestra) performances, in which groups would try to play at a superior level to their rivals 
(Jampadaeng et al., 2008, p. 127). Aside from this, evidence has been found regarding Piphat Mon musical performances during the reign of King Rama IV. A Piphat Mon performance was used at the funeral of Somdet Phra Debsirindramat, with her widower, King Rama IV, acknowledging her Mon ancestry. Following this funeral, Piphat Mon was accepted as a performance for funerals of the aristocracy, before gradually being used by people of all statuses (Banjun, 2008, p. 84-85).

In this era of globalization, there is a lack of traditional Thai musicians due to hurried surrounding conditions and poor economic climate, which cause people to have less time and interest in practicing Thai music. Additionally, Thai music is a difficult art to master and there is only a small number of living teachers with the required expertise to pass on the knowledge to younger generations. Some ensembles perform as they see fit, without adhering to the strict form of the art. For these different reasons, the number of traditional Thai musicians has decreased. One further factor that greatly affects the inheritance of traditional Thai music is changes to perceptions of popular music. Western music has exciting, fun rhythms and is easy to play. Traditional Thai music loses its audience to the popularity of Western music. Nowadays, at many auspicious and merry activities, such as marriage, birthdays and ordination ceremonies, people favor ensemble musical bands, known as Wong String, or string bands. These bands mostly play in Thai Derm or Thai Sakon rhythms, considering that traditional chanting when the bride and groom enter the marriage hall during wedding ceremonies is no longer popular. Most traditional Thai music ensembles or Wong are therefore only employed to play at events that are not joyful, notably funerals. These problems cause the economy of performers to suffer, cause some performers to change their career paths to alternative professions and have grave consequences for the inheritance and continuation of traditional Thai music (Tiyempipop, 1999, p. 121).

Apart from this, the transformation of economy, society, politics and culture in Thailand causes the lifestyle of Piphat Mon performers to gradually change, causes the harmony of musical customs and traditions and at the same time causes attitudes, beliefs and faith that have been upheld and inherited over time to slowly improve. However, the influence of western music culture has increased and Thai society has changed over time. As a result, a number of ceremonies have been lost. Piphat ensembles, which once had a role at these ceremonies, have seen their responsibilities in society reduced. The role of Piphat musicians and actors in making their own living must be adapted to current popular trends in society (Inkong, 2003, p. 2-3).

Nakhon Ratchasima, or Korat, is the largest area of Piphat Mon performing arts in the Northeastern region of Thailand (Isan). The province has similar social and cultural conditions to the central region of Thailand, allowing for the acceptance of Piphat Mon influence. Nowadays, Piphat Mon performances in Korat are experiencing artistic problems, including a lack of responsible work ethic, dishonesty amongst performers, and financial corruption. Additionally, there is a lot of Piphat Mon competition and some ensembles take work from others by charging reduced prices. This causes arguments, disagreements and poaching of performers.

Given the importance and current conditions of the various artistic and performing problems with Piphat Mon, the researchers were interested in studying the creation of performing arts standards in Piphat Mon in order to promote the Thai music profession in Korat lifestyle. This will increase knowledge of development methods of artistic performance, inheritance and management of music ensembles, including various customs of Piphat Mon performers, which will enable the promotion of progress in the Thai music profession. This will also enable performers to live a professional Thai music lifestyle in the current economic climate. Moreover, this study will publicize Thai music culture in Korat and promote the value of Thai culture for its continued existence.

\section{Research Aims}

This research has three main aims: a) to study the historical background of Piphat Mon artistic performance in Korat lifestyle; b) to study the current conditions of and problems with Piphat Mon artistic performance in Korat lifestyle; c) to create performing arts standards for Piphat Mon in Korat lifestyle in order to promote the Thai music profession.

\section{Research Methodology}

This study of Piphat Mon, creating performing arts standards in Korat lifestyle in order to promote the Thai music profession, is a research and development using a qualitative research method. Initial investigation was conducted through research and study of academic documents, books, concepts, theories and various related papers. The research area for this study comprised City District and Pak Chong District in Nakhon Ratchasima Province, Thailand. This area was home to six Piphat Mon ensembles: Udom Sin, Rattana Banleng, Pupirom, Sompot Tawornsiri, Thai Music Conservation Group Ban Pukao Lad, and Dong Banleng. These six ensembles perform Piphat Mon throughout the year. 
Data collection tools included basic survey, participant and non-participant observation, structured and non-structured interview and focus group discussion. Data collected from the survey, observation, interview and focus group discussion was checked for consistency. If any anomalies were discovered, the researchers returned to the research area to collect further data. The data was classified into groups according to the three aims of the research and the tools used for collection. Data was concluded by collection method.

Data analysis was conducted in line with a cultural qualitative research method using descriptive analysis technique. The data was verified and validated using both methodological and investigator triangulation methods.

\section{Research Results}

\subsection{The Historical Background of Piphat Mon Artistic Performance in Korat Lifestyle}

Piphat Mon performances were found to have existed in Korat for a long time and had a role in lullabies and for creating enjoyment in merit making, auspicious events and inauspicious activities. In the past, there was no light and sound equipment, so the performances had to rely on the musical sound of the Piphat and lullabies, as well as other performances, such as Thai Likay, which was a popular form of entertainment among Korat people.

This situation occurred until 1961, when teacher Ban Jantra, the head of an ancient Piphat ensemble, travelled to play music in Bangkok. This presented him with the opportunity to see Piphat Mon performances, which he found to be both extraordinary and beautiful. Jantra enjoyed the performance so much that he memorized the styles and characteristics, taking them back to his hometown when he returned from Bangkok. Upon his return, Jantra created two curved kong (gong cymbals) in the fashion of the Mon instruments in Ketdit style (whereby the hair of a monk is affixed to the curve of the cymbal). The deity Hanuman was engraved onto the instrument and the general style was in the Kankhot decorative pattern. In each instrument, thirteen small cymbals were arranged to complete the kong. The sound of the cymbals was comparable to Thai sounds and the instruments were played with a mixture of Thai and Piphat Mon styles. This was the birth of the first Piphat Mon instrument ensemble in Korat.

Prior to this event, during 1950-51, Mr. Bunyang Ketkong (a Likay teacher and performer of Piphat who would later become a national Likay performer in 1993) travelled from Bangkok to register as a student of teacher Tek Seausanga, a famous Likay instructor of the time. Ketkong was an extremely able Thai music teacher and inherited his ability from his elder brother Bunyong Ketkong (a national Thai musician in 1988). The two brothers practiced the art of Piphat music together throughout their childhood and came to know many famous Thai music teachers. Thus, when Bunyang Ketkong came to study under Tek Seausanga he brought with him a vast wealth of knowledge about the Piphat Mon style and incorporated this knowledge into his learning. This was the origin of knowledge transmission about Piphat Mon in Korat.

One further stage of the evolution of Mon music in Piphat Mon troupes in Korat was in 1969-1970, when the first Piphat Mon competition in Korat was held at the funeral of Luang Por Bun, Samorai Temple, City District, Nakhon Ratchasima Province. The competition was between the ensemble of teacher Yuan from Ban Kokpai, City District, Nakhon Ratchasima Province and the ensemble of teacher Cheung from Nakhon Si Ayutthaya Province. The ensemble of teacher Yuan used performers from the ensemble of teacher Jongkon Kaewpenka from Nonthaburi Province to help complete the music for his students. Teacher Somrit Kaewkemaim was sent to travel from Nonthaburi to help teacher Yuan. With the help of the Nonthaburi experts, teacher Yuan was able to resoundingly defeat teacher Cheung in the competition. This is another phase in the development of Piphat Mon artistic performance in Korat.

From 1998 until the present day, Korat society has changed. The popularity of Piphat Mon has declined and caused performers and heads of Piphat Mon ensembles to stop performing, sell their instruments to others, stop the transmission of knowledge and lose this precious heritage. Some students give up their study to pursue alternative careers, as the income from the Piphat Mon music profession is insufficient to cover daily living expenses. These changes have caused old Piphat Mon ensembles to alter their methods, sell their old instruments and develop new styles in line with current trends so that they are able to exist in modern society. There remain a number of ensembles in Korat who continue to play on a regular basis and enjoy fame in their profession. These are the ensembles of Udom Sin, Rattana Banleng, Pupirom, Sompot Tawornsiri, Thai Music Conservation Group Ban Pukao Lad, and Dong Banleng.

\subsection{The Current Conditions of and Problems with Piphat Mon Artistic Performance in Korat lifestyle}

\subsubsection{Current Conditions}

The current conditions were established from data collection via the methods of survey and interview about 
Piphat Mon performing arts in Korat. It was found that each ensemble will hold a ceremony to respect their teachers in the same fashion. This Wai Kru or Tam Gamnon ceremony involves the offering of fabric, flowers, candles, incense sticks and money to the instructors. Each ensemble will present the instructor with a Paan (a tray with a pedestal) with a white cloth upon it and the offerings will be placed upon the tray. The completed Paan will be placed at the front of the ensemble to respect the teacher each time the ensemble plays.

Only dedicated, unselfish performers with good behavior and a sense of responsibility will be selected to play in the ensembles. For the most part, only performers with a high level of knowledge and ability will be selected to perform. Instruments with a good condition and quality of sound will be selected. The characteristics of Mon instruments are beauty, engraving designs, gold inlays and mirrors. The faces of the monks and gods on the instruments must be beautiful and cheerful. The cymbals must have a good texture of casting. Each instrument must pass a tuning test to ensure harmony across the ensemble. There is a large number of Ranat Ek (Thai xylophone) made from the wood of both dalbergia oliveri and dalbergia cochinchinensis. However, the xylophones made from dalbergia oliveri are chosen for performances as their sound is clearer and more sonorous. For the Ranat Tum (a secondary Thai xylophone), bambusa nutans is chosen as the material, as it sounds better than other woods. For leather instruments, the fabric must be taut so that they produce a melodious sound when struck. Each instrument must compliment the others in the ensemble.

The information from the survey and interviews found that each ensemble is able to play the music required at ceremonies. These include temple music, local music, Cheun (invitation) music, Nang Hong music (used at funerals), fast music for Sangha (welcoming the monks to a ceremony), fast music for lighting candles at religious ceremonies, Mon funeral music, Faichum music and all general songs. Each performance will differ based upon the abilities of each ensemble. Each Piphat Mon ensemble in Korat organizes its instrument positioning differently but each adopts large gong ensemble stage positioning, in which the Mon gong is placed in the front row to the right of the ensemble and the Ranat $E k$ is in the row behind the gong. The Ranat Tum is placed to the left of the Ranat Ek but all other instrument positioning differs per group.

The head of the ensemble will organize the costumes for the performers. For the most part, performers will be given a white or black t-shirt to wear. In this way, the performers look neat. However, some ensembles do not give the performers specific clothing. In this case, the head will instruct the performers to wear shirts of the same color for the performance.

The performance will begin after the Wai Kru ritual has been performed to pay respects to the instructor. The first song in the performance will be the temple song. If the activity or event is not held in the temple, the ensembles will play the song of the host or a local song as the first piece, which compares to the form of traditional Thai music performances. Following this will be the cheun to respect the dead and performance of the nang hong will signal a break of ten to fifteen minutes. The production will continue with other Mon songs until the time of the funeral ceremony, when monks walk to recite the Abhidhamma or preach doctrine. The Piphat Mon group will play fast music to welcome the monks, during the lighting of candles, during worship of the three jewels, during breaks in the monks' ceremony and as guests are leaving the ceremony. On the day of the cremation, the Piphat Mon ensemble will play music during the funeral procession and the placing of the coffin on the cremation pedestal. During the cremation ceremony, the ensemble will play the temple or local song, followed by the nang hong to conclude the performance.

Most Piphat Mon ensembles in Korat do not have a raised stage or table for playing on, as in the central region of Thailand, which would incur additional costs and transportation difficulties. There are two ensembles that do use a raised stage and tables, namely Sompot Tawornsiri and Dong Banleng. This makes it more convenient as sometimes the locations of the performance are not suitable for the placing of musical instruments. They also use their own sound and lighting, which has proven favorable among audiences and has caused the income of the ensemble to be raised considerably.

The majority of Piphat Mon ensembles takes good care of and has covers for all of its instruments, especially the Mon gongs and xylophones. The instruments must all be protected, especially the gold gilding, mirrors and leather skins. Small instruments and sticks will be kept in wooden boxes. The adornments, such as peacock feathers, will be kept in boxes.

The ensembles will receive requests for work via telephone calls to their leaders. The price is agreed upon before performance and is based upon the size and length of the performance. The work is paid for by time. One performance means from the start of the event at about eight o'clock in the morning until four o'clock in the afternoon. If the event continues until eight o'clock in the evening, the host is charged for two performances in one day. The prices differ per event and per ensemble. This price depends upon many things, including musical 
instruments, skill required, staging, sound, lighting and transport. The price of each individual within the group is not equal. The wage is dependent upon ability, skill and the difficulty level of the instrument. For instance, the Pi Mon (a wind instrument) performers will receive a higher wage than other performers, no less than five hundred baht per performance. The ranat ek players will receive no less than four-hundred baht per performance, the gong players, ranat tum players and leather instrument players will earn between three-hundred and fifty and four hundred baht. The percussionists will earn about three hundred baht per performance. This also depends on the ability of the head of the ensemble to find work and will be reduced if the event cost is low.

Each ensemble will produce flyers or posters and affix them to the front of the instrument frames detailing the name of the group. Some ensembles will also include their address and contact telephone number. The ensemble also produces business cards to distribute to interested people. Aside from this, the ensembles give their information to the master of ceremonies to announce to the audience.

\subsubsection{Problems}

From the survey and interview, as well as the focus group discussion, it was discovered that artistic performances of Piphat Mon in Korat have a number of problems. Some performers are irresponsible and untrustworthy. This means that they accept work, yet do not appear to perform, causing the ensemble to be incomplete and resulting in problems for the head of the band. Some performers have problems with alcohol and cigarettes during the performance times. This causes a bad reputation for the entire ensemble. As well as this, some performers do not have suitable personal characteristics to play with the bands. Additionally, some performers boast of their own skills and obstruct the performances of others. This causes dissention and squabbles within the group.

Some ensembles have sound problems with their instruments, causing the overall sound of the performance to suffer. Sometimes, the metal components detach during performances and are unable to be fixed or replaced. Some troupes do not provide costumes for their performers and instead ask the performers to wear their own clothing. This causes the performance to look unprofessional. Piphat Mon groups have problems with their acting time and ceremony performances, which are caused by two factors. The first factor is that the performers do not possess sufficient intelligence in their performance to harmonize it with the ceremony and the second factor is that masters of ceremonies speak too much and do not give enough opportunity for the Piphat Mon ensembles to play.

The location is the problem that all troupes suffer with when they go to participate at a ceremony. Most places do not provide a suitable stage for the performance of Piphat Mon and do not allow a wide enough area for the ensemble. This causes the ensemble to sit in incorrect positions. Sometimes, there is also insufficient lighting for the performance. The host can become offended if the ensemble requests a change of location. It was also discovered that the heads of some groups accept work at prices that are too low, causing the wages of the performers to decrease. Occasionally, the ensembles are pushed out of work by a rival group offering a better deal. This causes disagreements between the ensemble leaders. Additionally some heads are not trustworthy and do not provide the agreed upon number of instruments or performers. This causes a bad reputation for the ensemble and is referred to as 'instrument deceit'. The heads of some groups pay reduced wages to the performers and do not pay on time. This causes the performers to lose faith in their art and occasionally to defect to rival ensembles.

\subsection{Creation of Performing Arts Standards for Piphat Mon in Korat Lifestyle in Order to Promote the Thai Music Profession}

The first standard is the standard of practice, comprising three elements of identification, which are pre-performance practice, performance practice and post-performance practice. Specifically, each ensemble must perform a Wai Kru ritual before each performance, must not speak, interact or walk around during the performance, must not lift their feet higher than the musical instruments, must not place items such as food or drink on the instruments, and must respect the instruments and their colleagues after the performance by way of a traditional Thai wai to show thanks and a professional apology for any mistakes made.

The second standard is the standard of performers, comprising three elements. The first element is that performers must have suitable and good personal characteristics, including readiness in performance and correct seating posture. The second element is that the performer must have a high skill and must be able to play all important musical pieces at different pace and rhythm levels, so that the ensemble remains in harmony. The third element is the required characteristics of a musician, including punctuality, abstinence from alcohol and cigarettes during performance, good human qualities, trustworthiness and responsibility in work and cooperation.

The third standard is the standard of instruments, comprising three elements. The first element is a complete set 
of musical instruments, according to the required amount stipulated by the host. The second element is the readiness of the instrument, which means that all instruments must be in the correct state for use. If there are any problems with the instruments, they must be fixed. The third element is the quality of musical sound. The instruments must all pass a tuning test and fit harmoniously with other instruments in the ensemble. Leather instruments must be taut.

The fourth standard is the standard of costumes, comprising two elements. The first element is that the uniforms must be a tidy match and that performers must wear long trousers. The costumes must be suitable for performance and not so tight as to restrict movement. The second element is that the hair of the performers must be cut neatly. Women with long hair must tie it correctly so that it does not cover their face.

The fifth standard is the standard of performance, comprising six elements. The first element is that the music must be harmonious, follow the requirements and include all important components of the ceremony. In addition, the sound must fall or rise according to the appropriate place in the song. The second element is the readiness of performance. The performance must begin when the Wai Kru ritual is complete. The music must start and finish together and the ensemble must contain the complete number of performers and instruments. The third element is the performance time. The Piphat Mon performers must arrive for the performance at the agreed time, perform harmoniously, cooperate and work hard. The fourth element is the stage and performance area. The stage must be raised between fifty and seventy centimeters and there must be cloth to cover the instrument tables. If there is no raised stage, the performance area must not be too narrow; it must be clean and convenient. The fifth element is sufficient light. The performers must be able to see their instruments clearly during the performance. There must be neon lighting on the ground or spotlights to light up the entire ensemble. The sixth element is the sound of the performance, which must be clear. The number of microphones must correspond to the number of instruments and paired instruments must be allocated a minimum of two microphones. Special Piphat Mon ensembles must have a minimum of three microphones and a mixer must be provided to control the level of sound. Speakers must not be placed further than twenty meters from the ensemble.

The sixth standard is the standard of management, comprising three elements. The first element is the acceptance of work in a trustworthy manner. The price of the work must be suitable and appropriate for the task. If a time and date is agreed upon, it must be upheld. The number of instruments must correspond to the agreed figure during negotiations. Work must not be stolen from other Piphat Mon ensembles. The second element is pricing. A fair wage must be paid to the performers and it must be paid on time. The third element is information. Posters must be created to advertise the ensemble. Business cards must also be created to distribute to interested parties in order to make future contact easy and convenient.

From the analysis of these six standards, it was found that Piphat Mon artistic performance is a product of culture. When various factors change, for example economy, politics, administration, education, religion and belief, performing arts are affected. Therefore, there must be alteration of the form of performance in order to help the performing art and its performers survive in society. The creation of performing arts standards for Piphat Mon ensembles in Korat is one tool to ensure that the form of performance remains and develops to help promote the Thai music profession.

\section{Discussion}

\subsection{The Historical Background of Piphat Mon Artistic Performance in Korat Lifestyle}

From the study of documents and collection of field data, it was found that Piphat Mon musical ensembles have existed for a long time and have a role in Korat society. The research results correspond to the study of Kanok Klaimuk (1998, p. 22), which stated that the lifestyle of Thai people from the past to the present has been a lifestyle full of music because Thai nationals are a people who value enjoyment and merriment in their lives. People try to find fun and happiness in their lives. This is a lifestyle that is both good and complete. The attitudes of Thai people in the past involved belief in religion, which stated that people must make merit. This attitude began at birth and continued through childhood, ordination, marriage, anniversaries and birthdays, until death. Thai music was associated with ceremonies in each step of the life-cycle. Aside from this, Thai music was visible in Khon (a masked dance), dramas and Likay, for which Piphat ensembles were used to provide the musical accompaniment.

Piphat Mon is continually developed and musicians travel to the Central Region of Thailand to purchase new instruments and equipment. The Central region is the most prominent Piphat musical area in the country and has a clearly developed performance model for piphat mon khrueang khu (a medium size Piphat Mon ensemble). Piphat Mon is considered a music business, as well as a part of the business of managing funeral pyres and crematoriums at temples in Korat. From 1969 until the present day, Piphat Mon has been popular at funeral 
ceremonies of the rich in Korat. These findings adhere to the work of Jedcharin Jirasantitam (2010, pp.77-79), which stated that Piphat Mon ensembles are musical ensembles influenced by the Mon culture. The Mon people brought their musical instruments with them when they immigrated to Thailand. The style developed by incorporating the hard-wood instruments of existing Piphat ensembles in Thailand. The ensembles were gradually associated with funerals.

The person primarily responsible for the amalgamation of Thai and Mon styles was performance art teacher Luang Bradit Pairo. The Mon instruments are placed at the front of the ensemble, including the Mon gong, Mon flute, Mon two-faced drum and the Beung Mankok. Behind these are placed the two Thai xylophones and other musical instruments. Once the ensemble has been arranged, the differences are clear to see. Actually, the Piphat Mon ensemble is used by Mon people at both auspicious and inauspicious events, including celebration ceremonies of the Thonburi Jade Buddha and funerals. Thai people will only use the Mon ensemble for funerals, as the sound is considered melancholy and suitable to the atmosphere. There are three sizes of Piphat ensemble, which are piphat mon khrueang ha, piphat mon khrueang khu and piphat mon khrueang yai. These findings are corroborated by the research of Somsak Srisantisuk (2001, p. 96), which considered the dissemination of thought, belief, knowledge, ability, skill and expertise among relations, people with similar characteristics and close friends. The results showed that if people move from place to place, they will take their background with them and disseminate knowledge to new areas.

The ability of a host to employ a Piphat Mon ensemble at a funeral gives some indication of their social and particularly financial status. If a person employs an ensemble, the implication is that they are financially secure. In this way, Piphat Mon ensembles act as a symbol of class, corresponding to the research of Srisantisuk (2001, pp.18-80).

\subsection{The Current Conditions of and Problems with Piphat Mon Artistic Performance in Korat Lifestyle}

The research results regarding the uniformity of the Wai Kru ritual prior to each performance agree with the findings of Suradit Paksuchon (2007, p. 61). Paksuchon studied the offering procedure of the ritual and found that once the ensemble was prepared, the head of the ensemble must place the offering and pay respects to the instructors, sacred objects and various guardian spirits for luck and prosperity before the performance. This also agrees with the results of Chuchat Pinpat (2003, p. 173-189), which discussed the beliefs and customs of performers in Piphat Mon ensembles. The ritual of Wai Kru must include flowers, candles, incense sticks, money (specifically six baht) and one bottle of Thai rice whisky.

The results concerning the order of the ceremony correspond to the research of Chaloemsak Pigunsri (1996, pp.137-139), which concern the use of Mon songs in Piphat Mon performances. Pigunsri discovered that the Cheun music to invite the spirits is in two forms, small and large. The characteristics and style of this music is similar to the home rong music of Thai Piphat ensembles and has meaning for the gods and sacred objects. The music also has two aims. The first is to pay respect to the gods and holy objects that Mon people venerate and the second is to respect the spirit of the deceased at the funeral. The temple music is used to announce broadcasts when there are no ceremonies. When funeral ceremonies are held at the temple, this music is used to announce the event and can be compared to nang hong music in a traditional nang hong ensemble. The local song has a similar melody and rhythm to the temple song but the start of the music is different. This music is used to announce the occurrence of a funeral ceremony to the local people. The local song can be played on two occasions at the funeral, which are at the end of a performance in the evening and during the funeral ceremony. Music played in the evening (Pleng Yamkam) is often from long compositions and can be classified to three rhythm grades, which are slow, medium and fast. These three grades are similar to the three levels used in Thai Pleng Tao music. However, there are some differences between Pleng Yamkam and Pleng Tao. Pleng Yamkam is played at nightfall before the Abhidhamma ceremony, as it is long. The music takes approximately one hour to complete and its completion will correspond with the beginning of the Abhidhamma ceremony. The prominent characteristic of Pleng Yamkam is a single piece of music that incorporates each instrument in the ensemble in slow, medium and fast rhythms. This means that each performer must play at three paces during the performance. Music played in the early morning (Pleng Yamrung) incorporates two styles. If the ceremony is at a temple, the ensemble will play the temple music, but if the ceremony is held externally, the ensemble will play local music. Afternoon music (Pleng Yamtieng) is played between approximately half past twelve and half past one in the afternoon. Many songs are played in the same performance. At the end of each piece of music, techniques will be used to break the rhythm of the music so that each piece is played independently. It is also popular to use a form of dancing during the afternoon performance, entitled Ram Mon. Music used in the funeral procession is a collection of pieces of music that have many different segments. Each segment will be short and have similar key characteristics to cheud music that is played in traditional hard-wood Piphat ensembles. Each segment will 
have the same ending melody in the rondo form. The music is played while the corpse is being moved from one location to another. Faichum music is the final style of composition and is used during the cremation ceremony as it is short.

The financial aspects of the results correspond to the research of Yotsapan Demyaem (2003, p. 96-98), which concerned the music culture of Lopburi. The investigation found that the management of all Thai music ensembles in Lopburi was similar. The head of the ensemble would be responsible for covering the costs of accommodation, food and other necessities, in addition to the transmission of knowledge about Thai music performance and the maintenance and care of the instruments. With regards finding work, there were found to be two methods; the host either visits the ensemble leader's home or workplace or contacts the ensemble leader by telephone, which is an important form of communication for modern ensembles. Aside from this it was found that there were three types of work, which were religious ceremonies, life-cycle ceremonies and performances incorporating dancers.

\subsection{Creation of Performing Arts Standards for Piphat Mon in Korat Lifestyle in Order to Promote the Thai Music Profession}

From the analysis of data, six standards, with a total of twenty elements were identified. The first standard of practice corresponds to the research of Montri Dramote (1999, p. 71), which discussed the purpose of the Wai $\mathrm{Kru}$ ritual and found that the correct performance of the ritual has many benefits. These benefits are the maintenance of Thai customs, the setting of good behavioral examples and respect for future generations to follow, the safeguarding of prosperity and fortune, and the creation of unity within the ensemble. The second standard (of performers) agrees with the findings of Chuchat Pinpat (2003, p. 173-189). Pinpat concluded that there are a number of obligatory actions that the performers must all perform: a) carry out the ritual of Wai Kru prior to performance; b) lift all sticks in the air before performance; c) refrain from tapping the instruments during breaks in performance; d) prohibit all spectators from playing with the instruments; e) refrain from walking above or over the instruments; f) point feet away from the instruments; g) sit below the instruments; $h$ ) pay respects to the instruments after performance, in the form of a traditional wai; i) refrain from passing or accepting objects over the instruments. The sixth standard (of management) corresponds to the work of Suparat Tongpanit (2012). Tongpanit studied the creation of artistic performance standards of music in Korat, Nakhon Ratchasima and identified seven standards, which were: a) performers; b) lyrics and language; c) dance postures; d) costume; e) stage, light and sound; f) performance time; g) performance conventions. From the results of the study it can be concluded that the standards of Korat music can help the audience to understand the performances and can help the performances represent the local identity. The performances use postures and instruments, as well as emotion, language and sound to show the quality of the customs. They have an organized plan and uniform standards to ensure that the art-form is developed and systematically recorded. Each ensemble has a system and uses the same model, which allows it to present a clear portrayal of the local identity, without disregard of its history.

\section{References}

Banjung, O. (2008). Memorials of Thai-Mon people making merit in dedication to Galyani Vadhana, the Princess of Naradhiwas, according to traditional Mon beliefs. Bangkok: October Printing.

Demyaem, Y. (2003). Study of music culture in City District, Lop Buri Province. Bangkok, Srinakharinwirot University.

Dramote, M. (1999). 'Turiyasan', the obituary of Mr. Montri Dramote. Bangkok: Pathum Thani University.

Inkong, R. (2003). Inheritance of piphat in Ang Thong Province. Bangkok: Srinakharinwirot University.

Jampadaeng et al. (2008). Method of conservation and inheritance of Piphat ensembles in Ban Maw, Kewa Sub-District, City District, Mahasarakham Province. Mahasarakham: Mahasarakham University.

Jirasantitam, J. (2010). Theories of Thai singing. Bangkok: Odeon Store.

Klaimuk, K. (1998). Transmission of piphat in the districts of Nakhon Sri Ayutthaya. Bangkok: Mahidol University.

Paksuchon, S. (2007). Harmonious music lineage of piphat mon, City District, Pathum Thani Province. Bangkok: Kasetsart University.

Pigunsri, C. (1996). Culture of piphat mon in Thailand. Thai Music Higher Education No. 27. Chonburi: Chonburi University

Pinpat, C. (2003). Study of piphat mon culture, City District, Pathum Thani Province. Bangkok: Srinakharinwirot 
University.

Srisantisuk, S. (2001). Study of society and culture: thought, methodology and theories. Khon Kaen: Ammarin Copy.

Tiyempipop, C. (1999). Conservation and inheritance of Thai music in Nakhon Nayok Province. In Thai Music Higher Education No. 31. Nakhon Nayok: Chulachomklao Royal Military Academy.

Tongpanit, S. (2012). Korat music: creation of local performing art standards in Nakhon Ratchasima. Mahasarakham: Mahasarakham University.

\section{Copyrights}

Copyright for this article is retained by the author(s), with first publication rights granted to the journal.

This is an open-access article distributed under the terms and conditions of the Creative Commons Attribution license (http://creativecommons.org/licenses/by/3.0/). 